\title{
Flow Structures of Submerged Gas Jet in Liquid Currents
}

\author{
Ping Dong ${ }^{1,2 *}$, Dong Cheng ${ }^{1,2}$, Huixiang Jing ${ }^{1,2}$, Guanghua $\mathrm{Li}^{1,2}$, Bingju $\mathrm{Lu}^{1,2}$ and Ximeng \\ Wang ${ }^{1,2}$ \\ ${ }^{1}$ The 713 Research Institute of CSSC, ZhengZhou 450000, China \\ ${ }^{2}$ Henan Key Laboratory of Underwater Intelligent Equipment, ZhengZhou 450000, China
}

\begin{abstract}
The flow structure of the submerged gas jet in liquid currents is important to engineering applications. In the present study, the development of a submerged gas jet subjected to liquid current is experimentally investigated to evaluate the effects of the current on the underwater gas jet evolution. A full-scale experimental setup is designed for submerged gas jet release and dispersion in the liquid currents with different velocities. The flow structures of the gas jet are captured by shadow photography combined with a high speed video camera. The experimental images are processed to extract the parameters and perform Proper Orthogonal Decomposition (POD) analysis to reveal the characteristics of different modes standing for different flow structures. It turns out that the flow structures of the gas jets submerged in liquid currents with different velocities are affected by the liquid currents and gas jet pulsation, and the analysis will provide credible assessment and opportunity to take prompt response to control potential accidents caused by the submerged gas jet release in liquid current.
\end{abstract}

\section{Introduction}

The submerged gas jet in liquid currents is a classical complicated and unsteady multiphase flow, and it has become increasingly significant in many areas of scientific and engineering research such as underwater vehicles drag reduction where the flow pattern of the gas jet is greatly concerned[1,2], and water piercing launch (WPL), where the successful launch of the under missile depends critically on the launch path formed by the gas jet[3], and the chemical industry where the mixing induced by the gas jet promotes chemical reactions[4]. In natural gas engineering, a deep comprehension on jet evolution in the liquid cross currents would help to predict the gas leakages[5,6]. The underwater gas jet in quiescent liquid has been widely studied, and theoretical and numerical analysis has been proposed by the former researchers. However, limited information on the gas jet submerged in liquid cross flow appears in the available scientific literature, especially on experimental research.

As a gas jet is injected into the liquid currents, a sophisticated flow structure is formed. These flow structures contain a jet regime region and a quasi buoyancy regime region

\footnotetext{
* Corresponding author :dongping@mail.ustc.edu.cn
} 
jointed by a transition region as indicated in the former researches[7,8]. Across the first regime region, the flow characteristics of the jet are dominated by the initial gas momentum and the liquid currents. In the quasi buoyancy regime region, the buoyancy and the liquid current determine the flow pattern, and a column of slopping bubbles are formed as the jet reaches some distance from the nozzle exit. A quantity of studies adopting experimental and numerical methods have been conducted to figure out the nature of the submerged gas jet and its flow characteristics[9-11]. Weiland and Vlachos studied the roles of independent parameters in determining the gas jet flow regimes, and inferred that the gas jet flow regimes depend on the Mach number at the nozzle exit[12]. Computation fluid dynamics is a pervasive numerical approach for the gas jet in liquid currents, and flow structures of the submerged gas jet are described in the former studies[13]. With the consideration of a variety of necessary physics such as non-ideal gas behavior and Lagrangian approach, and assumptions that the gas jet velocity and distribution can be represented by the Gaussian or top-hat functions, a series of integral models were developed and verified for submerged gas jets in different situations containing horizontal and vertical directions[14].

The aim of this paper is to analyse the submerged gas release and dispersion in liquid current with different velocities by experimental approach. A full-scale experimental system is designed to carry out experiments under different liquid current velocities. The POD method is adopted to extract the flow structures of the gas jets submerged in liquid currents with different velocities, and the mode energy is analysed.

\section{Experimental method}

The experiments were carried out by a full-scale experimental system in the physical model basin of the marine laboratory in the 713 institute of CSSC. Three parts including the experimental basin, the movable injector system and the imaging system forms the main frame of the experimental setup, and the details of the experimental system can be found in Figure.1.

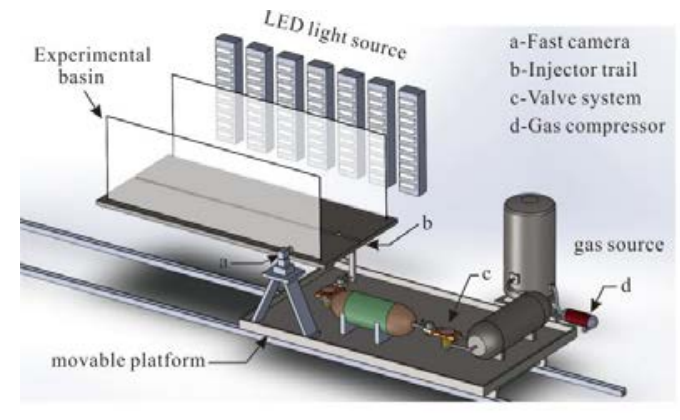

(a)

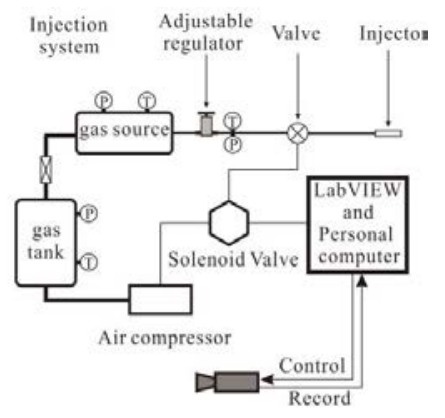

(b)

Fig. 1. Experimental setup and subsystem.

The gas-injection system which is a key point to the experimental implementation is elaborately designed. A constant mass flow which is delivered to the injector was realized by adopting a gas source combining with a gas pressure regulator that is insensitive to backpressure changes in the ambient. The mass flow rate delivered to the injector was calculated according to the ideal gas equation by monitoring the pressure and temperature of the gas source. Desired Mach number was achieved by adopting different nozzles which were manufactured by the $3 \mathrm{D}$ printing technology with a constant exit diameter and a varying throat diameters. In the present study, the gas source adopted dried air as the experimental gas, and the liquid currents are formed by tap water. The injector system 
locates beneath the bottom of the basin and can move stably along the center line of the basin. The initial gas jet and ambient liquid currents are formed when the gas is released from the injector which is moving along the basin center through a device. The experimental basin is $80 \mathrm{~m}$ in length and $6.5 \mathrm{~m}$ in width and $12 \mathrm{~m}$ in depth. The ambient pressure at the nozzle exit can be changed according to adjust the depth of the initial experimental water depth (the depth of the water in the present is sustained at $10 \mathrm{~m}$ ). When the experiments are carried out, submerged gas jet is injected into the basin and the injector moves at a fixed initial velocity. The system was controlled by LabVIEW software and a data acquisition system.

Table 1. The initial experimental parameters.

\begin{tabular}{|c|c|c|}
\hline Parameters & Value & Description \\
\hline $\mathrm{U}_{\infty}$ & $0.3 \mathrm{~m} / \mathrm{s}$ to $2 \mathrm{~m} / \mathrm{s}$ & Velocity of the liquid current \\
\hline $\mathrm{m}_{\mathrm{i}}$ & $0.17 \mathrm{~kg} / \mathrm{s}$ & Mass flow rate \\
\hline $\mathrm{t}$ & $5 \mathrm{~ms}$ & Exposure time \\
\hline $\mathrm{d}_{\mathrm{N}}$ & $12 \mathrm{~mm}$ & Nozzle diameter \\
\hline
\end{tabular}

The details of the imaging system which consists of a high speed camera (Photron FASTCAM APS-RX in conjunction and a Canon lens (EF 24-30 mm, f/28L II USM)) and illumination are depicted in Figure.1(b). The imaging system was fixed with the injection system and the flow morphology of the gas jet are recorded through the test section. All the experiments were recorded for $5 \mathrm{~s}$, and the test section were recorded with $1024 \times 860$ pixel resolution. The details of the initial experimental conditions are listed in Table 1.

\section{Results and discussion}

Experiments with different initial conditions were performed to study the evolution of gas jets injected into liquid currents with different velocities. The flow characteristics of each case were achieved from the experimental results, and by a method used in our former work[9] the raw images were processed to extract the flow structures.

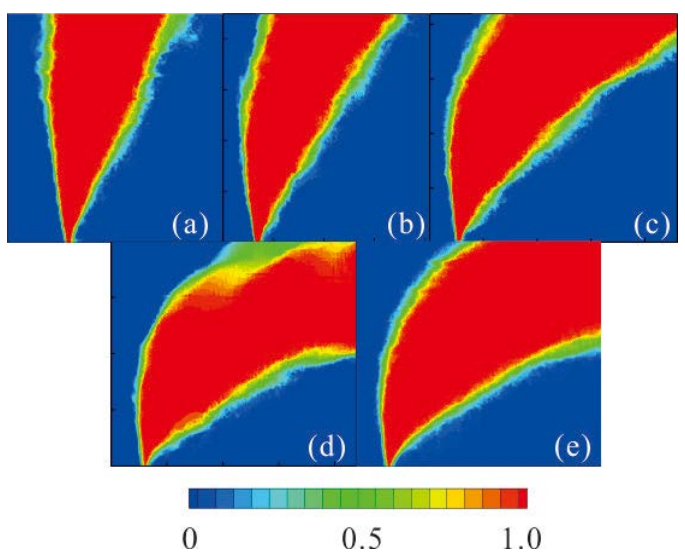

Fig. 2. Time average results of submerged gas jets in different liquid currents, with velocity of (a) $0.35 \mathrm{~m} / \mathrm{s}$, (b) $0.7 \mathrm{~m} / \mathrm{s}$, (c) $1.0 \mathrm{~m} / \mathrm{s}$, (d) $1.5 \mathrm{~m} / \mathrm{s}$, (e) $2.0 \mathrm{~m} / \mathrm{s}$ 


\subsection{Flow morphology}

The summation results of the gas jets in liquid currents are depicted in Figure.2. The velocities of the liquid currents vary from $0.35 \mathrm{~m} / \mathrm{s}$ to $2 \mathrm{~m} / \mathrm{s}$. It can be seen from the figure that flow structures and the developing of the gas jet in liquid currents are essentially unsteady and turbulent. There are appreciable fluctuations emerging on the gas liquid interface. During the gas jet evolution, the gas momentum determines the jet flow morphology, and the effects of the liquid current relying on the its velocity magnitude. As depicted in Figure.2(a), the gas jet develops with few effects from the current for the small magnitude of the liquid current velocity. The boundaries between the gas jets and liquid current bend more and the fluctuations intensified as the liquid current velocity increases.

\subsection{POD analysis}

The Proper Orthogonal Decomposition (POD) was introduced in the context of fluid mechanics. In the present study, the so-called snapshot POD proposed by Sirovich et al[15] is used to extract the coherent structures on the interface between the gas jet and liquid currents.
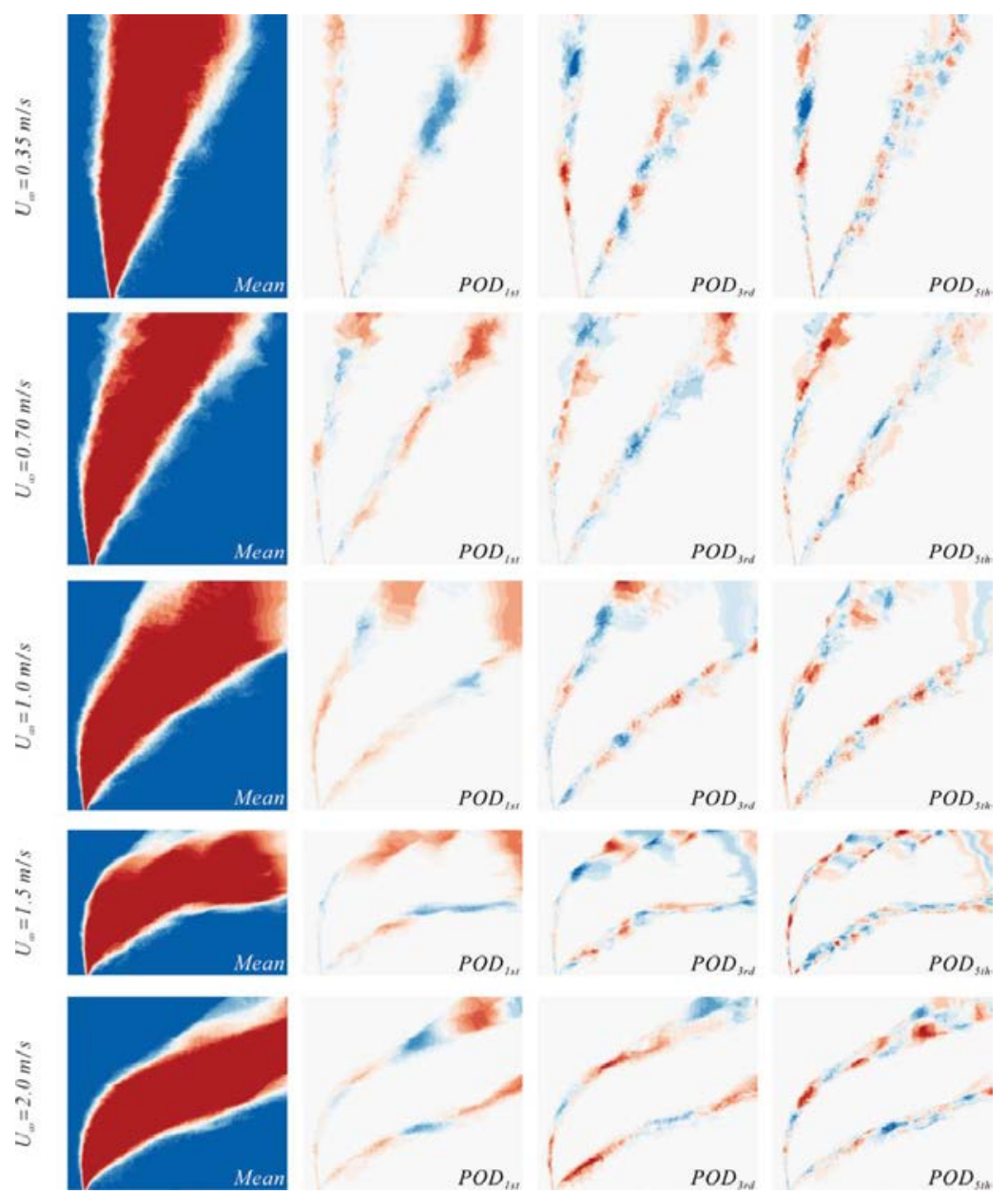

Fig. 3. The average results and POD modes for cases with different liquid currents. 
To express multidimensional data sets with a reduced number of dimensions, the snapshot POD is always adopted as a method. In fluid mechanics, the application of the POD is well established for analysis on the vector fields, and the dominant modes or coherent structures can be extracted, while it has been seldom applied to the scalar field. Applications of POD method on the flow structures of liquid jet in gaseous current have been demonstrated in several researches[16,17], while for the submerged gas jet in liquid current few researches have been published.

In the present study, data sets of 800 (sufficient to perform POD analysis) shadowgraph images were analyzed (i.e. the number of sequences $\mathrm{N}=800$ ), and the corresponding POD field of views are the same as the processed shadowgraph in section 3.1. The dominant features and coherent structures can be identified and extracted from the field as the submerged gas jet was released in the liquid current. The processed binary sequences which are used to achieve the summation results were analyzed with the snapshot POD for the orthogonal decomposition of flow information.

According to the snapshot POD, the orthogonal basis functions $\Phi$ and time dependent orthogonal amplitude coefficients (temporal coefficients) $a_{r, i}$ at time $t_{i}(i=1,2, \ldots, N)$ can be extracted, and thus the flow property $(f(x, t))$ can be expressed by the first $M(M \leq N)$ modes as:

$$
f(x, t)=\bar{f}(x)+\sum_{r=1}^{M} a_{r, i} \phi_{r}
$$

The mean, and first, third and fifth modes for cases with different current velocities are depicted in Figure.3. Due to the unsteady and unstable nature of the submerged gas jet, the information of the coherent structures could not be detected over the mean flow field. Instead, as shown in Figure.3, coherent structures can be identified over different modes. Comparing the first mode of POD with different experimental sequences, it can be stated that the first mode clearly represents the bag structure caused by the submerged gas jet pulsation.

\subsection{Mode energy}

To reveal the energy level of different POD modes, the energy levels of different modes for the case with $1.5 \mathrm{~m} / \mathrm{s}$ liquid current are depicted in Figure.4. It can be seen from Figure.4, the energy level of the first mode is over 35\%, suggesting that the pulsation of the submerged gas jet dominates the flow fluctuation.
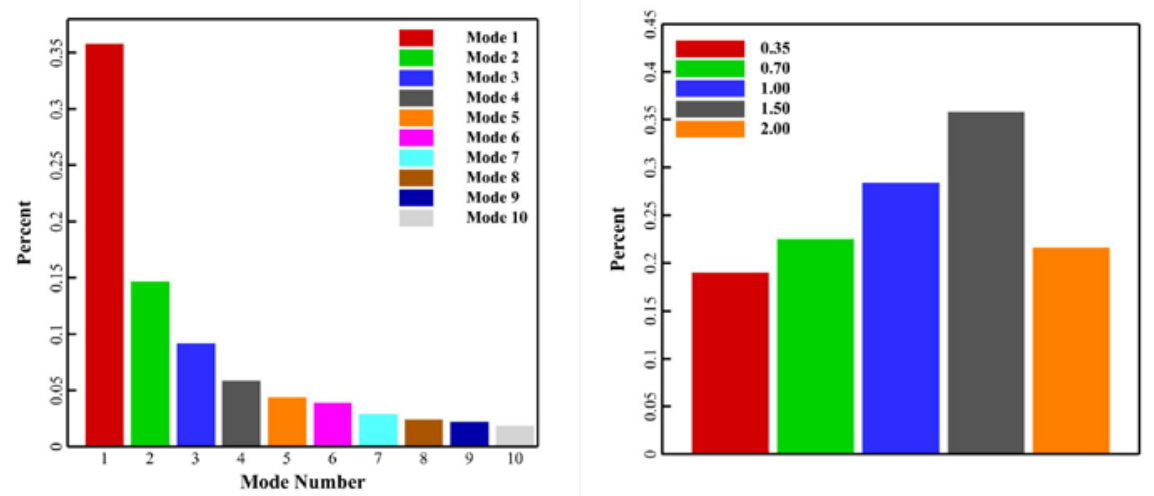

Fig. 4. Energy levels of the first 10 modes for the case with the liquid current of $1.5 \mathrm{~m} / \mathrm{s}$ (left), and the effects of the currents on 1st mode energy distribution, the unit of the legend is $\mathrm{m} / \mathrm{s}$. 
The energy levels of the first mode for all the cases are also depicted in Figure. 4. It is found that the energy level of the first mode is indeed a non-monotone function of liquid current velocity. According to the former analysis, the first mode is affected by the gas jet pulsation, which depends on the liquid current velocity and the initial gas jet characteristics. With the increase of the current velocity, the pulsation increases for high pressure at the nozzle exit formed by the current, while for the case with $2.0 \mathrm{~m} / \mathrm{s}$ velocity, the magnitude of the current velocity is large enough to suppress the pulsation of the gas jet, and correspondingly leads to the decrease of the first mode energy level.

\section{Conclusion}

The submerged gas jet in liquid current was experimentally investigated by adopting a fullscale experimental system. The POD method was adopted to perform analysis on the processed submerged experimental sequences to analyse the flow structures of the gas jet and the effects of the liquid current. The results from experiments are valuable for describing the submerged gas jet, which could provide accurate initial parameters for the subsequent gas jet and plume evolution. The present study provides a deep insight into the behavior of the submerged gas jet in liquid current.

This work was financially supported by the Natural Science Foundation of Henan Province (Grant No. 202300410003). The support from Dr. Fu is gratefully acknowledged.

\section{References}

[1] P.A. Brandner, B.W. Pearce, K.L.de. Graaf, J. Fluid Mech. 768, 141-174 (2015)

[2] S.L. Ceccio, Annu. Rev. Fluid Mech. 42, 183-203 (2010)

[3] X. Zhang, Y. Yu, L. Zhou, Int. J. Heat Mass. Tran. 134, 250-261 (2019)

[4] S.S. Gulawani, S.S. Deshpande, J.B. Joshi, M.S. Shah, C.S.R. Prasad, D.S. Shukla, Ind. Eng. Chem. Res. 46, 3188-3218 (2007)

[5] Z. Geng, X. Li, G. Chen, H. Zhu, S. Jiang, Process. Saf. Environ. Prot. 149, 11-21 (2020)

[6] X.H. Li, G.M. Chen, F. Khan, J. Hazard. Mater. 367, 646-685 (2019)

[7] H. Shi, Q. Guo, C. Wang, R. Dong, L. Zhang, H. Jia, X. Wang, B. Wang, Shock Waves 20, 347-352 (2010)

[8] P. Dong, B. J. Lu, S. F. Gong, D. Cheng, Exp. Therm. Fluid. Sci. 112, 109998 (2020)

[9] T. I. Mohamad, Fuel 160, 386392 (2015)

[10] D. Chong, Q. Zhao, F. Yuan, Y. Cong, W. Chen, J. Yan, Exp. Therm. Fluid. Sci. 68, 744-758 (2015)

[11] Q. Xu, L. Guo, L. Chang, Y. Wang, Int. J. Heat Mass Transfer 103, 305-318 (2016)

[12] C. Weiland, P. P. Vlachos, Int. J. Multiphase Flow 48, 46-57 (2013)

[13] L. Liu, O. Keplinger, T. Ma, T. Ziegenhein, N. Shevchenko, S. Eckert, H. Yan, D. Lucas, Chem. Eng. Sci. 192, 288-305 (2018).

[14] J. Ma, Y. Song, P. Zhou, W. Cheng, S. Chu, Exp. Therm. Fluid Sci. 92, 1-11 (2017)

[15] L. Sirovich, Q. Appl. Math. 45, 561-571 (1987)

[16] K. E. Meyer, J. M. Pedersen, O. Zcan, J. Fluid Mech. 583, 199-227 (2007)

[17] M. Broumand, M. Birouk, S. V. Mahmoodi J, J. Fluid Mech. 879, 775-792 (2019) 